\title{
Value-Based Insurance Design: Aligning Incentives, Benefits, and Evidence in Oncology
}

Jonas A. de Souza, MDa; Mark J. Ratain, MDª, and A. Mark Fendrick, MD, Chicago, Illinois and Ann Arbor, Michigan

\author{
Key Words \\ Cost-sharing, economics, cost-benefit analysis, neoplasms, health \\ care reform, reimbursement mechanisms
}

\begin{abstract}
When everyone is required to pay the same out-of-pocket amount for oncology services for which benefits depend on patient characteristics and clinical indication, there is enormous potential for both under- and overutilization. Unlike most current health plan designs, the value-based insurance design (V-BID) explicitly acknowledges and responds to patient heterogeneity across the entire continuum of cancer care. By adding "clinical nuance" to benefit design, V-BID encourages the use of services when the clinical benefits exceed the cost, and likewise discourages the use of services when the benefits do not justify the expenditure. This manuscript further describes the concept of V-BID, creates a framework for its development in oncology, and outlines how this concept aligns with ongoing research, care delivery, and payment reform initiatives. (JNCCN 2012;10:18-23)
\end{abstract}

Medical science has made great progress in enabling the ability to successfully prevent, diagnose, and treat cancer. Successful interventions include human papillomavirus vaccine, colorectal cancer screening, and treatments such as imatinib, which changed the natu-

From asection of Hematology/Oncology, Department of Medicine, The University of Chicago, Chicago, Illinois; ${ }^{b}$ Comprehensive Cancer Center and Committee on Clinical Pharmacology and Pharmacogenomics and Center for Personalized Therapeutics, The University of Chicago, Chicago, Illinois; and 'Department of Internal Medicine and Health Management \& Policy and Center for Value-Based Insurance Design, University of Michigan Medical School, Ann Arbor, Michigan.

Submitted October 25, 2011; accepted for publication October 31, 2011.

The authors have disclosed that they have no financial interests, arrangements, or affiliations with the manufacturers of any products discussed in this article or their competitors.

Correspondence: Jonas A. de Souza, MD, The University of Chicago Medical Center, 5841 South Maryland Avenue, MC 2115, Chicago,

IL 60637-1470. E-mail: jdesouza@medicine.bsd.uchicago.edu ral history of chronic myelogenous leukemia (CML). Nevertheless, cancer care has also become increasingly unaffordable to many patients. The National Health Interview Survey reported that more than 2 million cancer survivors in the United States did not get one or more needed medical services because of financial concerns. ${ }^{1}$ Another study showed that $13 \%$ of people with cancer spend at least a fifth of their income on health care and insurance. ${ }^{2}$ Furthermore, in current insurance designs, this financial burden to the patient is irrespective of the quality of life and survival benefit a specific intervention provides. In this archaic "one size fits all" approach, the heterogeneity between highand low-value interventions is not appreciated at all, in that they bear the same financial burden to the patient, regardless of clinical benefit.

With the goal of adding "clinical nuance" to benefit design, and therefore selectively improving access to high-value interventions, section 2713(c) of the Patient Protection and Affordable Care Act (PPACA) included a concept that allows guidelines development using value-based insurance designs (V-BIDs). V-BID-the basic premise of which is to reduce barriers to high-value medical services-has been increasingly implemented in the management of chronic diseases, such as heart disease, diabetes mellitus, and asthma. The most commonly implemented V-BID programs lower copayments on classes of medications identified as high value. For example, the program run by Pitney Bowes provides asthma and diabetes medications to its employees for free, and the company reported a 19\% decrease in costs per asthma patient. ${ }^{3}$ Other programs, such as the Asheville Project, target patients with a particular clinical condition. The Asheville Project waives copayments for diabetes care for Asheville city employees who meet with a trained pharmacist. The program reportedly improved economic and health outcomes over 5 years. Al- 
though prescription costs rose, overall medical costs declined, and patients' glycosylated hemoglobin and lipid levels improved. ${ }^{4}$

Given the evidence-based quality metrics in cancer care, and the well-described quality gaps, it has become incumbent on the delivery system to create mechanisms for patients and providers to enhance quality and constrain costs. This brief report describes the concept of V-BID and its potential role in oncology, and further argues that it promotes 3 important aspects of oncology care: access to highvalue interventions, evidence-based use, and personalized medicine.

\section{Applying V-BID to Health Care Interventions in Oncology}

The Agency for Healthcare Research and Quality defines health care intervention as "any type of treatment, preventive care, or test that a person could take or undergo to improve health or to help with a particular problem. Health care interventions include drugs..., foods, supplements..., vaccinations, screening tests..., exercises..., hospital treatment, and certain kinds of care...." Along this line, V-BID seeks to align the out-of-pocket cost of a health care intervention with the benefit it provides through the entire continuum of cancer care, from prevention and early diagnosis to curative or palliative treatment. Implementing V-BID programs in oncology would be founded on 4 principles: 1) nonselective patient cost-sharing programs based exclusively on price can discourage use of high-value, potentially life-saving interventions; 2 ) the clinical benefit that interventions provide differ quantitatively and qualitatively, depending on indication and clinical setting (e.g., adjuvant vs. palliative, or survival vs. symptom improvement); 3 ) the levels of evidence supporting interventions differ, and although an intervention may have irrefutable survival benefit in a specific scenario, it may lack evidence for other indications; and 4) V-BID programs acknowledge patient population heterogeneity and promote personalized medicine, as the value of a specific intervention varies across patient groups, and biomarkers may identify those who would benefit most.

\section{Overarching Goal: Improve Access to High-Value Interventions in Oncology}

Out-of-pocket costs for oncology patients have risen at a much higher rate than health plan costs, ${ }^{6}$ and high-deductible consumer-directed plans are becoming more common. ${ }^{7}$ However, for example, switching to a high-deductible health plan was recently associated with a downward trend in colorectal cancer screening after 2 years on the plan. ${ }^{8}$ Colorectal cancer is the third most commonly diagnosed malignancy in the United States, with approximately 110,000 new cases annually. ${ }^{9}$ Its incidence and mortality rates have been decreasing in the United States over the past 2 decades, ${ }^{9}$ and colorectal cancer screening has been successful in diagnosing early-stage and curable cancers and for detecting and removing adenomatous polyps. Further, some evidence suggests that colorectal cancer screening is underused in the Unites States. ${ }^{10}$ Therefore, focusing on the continuum of cancer care, the California Public Employees' Retirement System (CalPERS), the nation's largest nonfederal purchaser of health benefits, established a $\mathrm{V}$-BID program to promote colonoscopy in preferred clinical settings. ${ }^{11}$

Although traditionally performed in hospital outpatient centers, colonoscopies are increasingly being performed at ambulatory surgery centers. Specifically, CalPERS instituted a $\$ 0$ copayment for Blue Shield members for colonoscopies performed at in-network ambulatory surgery centers and a $\$ 250$ copayment for those who select an in-network outpatient hospital. CalPERS is thus applying a V-BID program to shift procedures from higher-cost hospital outpatient centers to lower-cost ambulatory settings. Beyond this application, this approach to V-BID could possibly be used to shift oncology patients to institutions with reported greater quality measures, higher guideline-adherence rates, or preferred providers.

Further, a database analysis of pharmacy claims paid by private insurers and Medicare showed that patients with cancer with copayments greater than $\$ 500$ were 4 times more likely to abandon treatment than those with copayments of $\$ 100$ or less. ${ }^{12}$ This underscores the financial burden patients must overcome to obtain cancer care. As an example, the targeted agent imatinib has changed the natural history of CML. It gained approval as standard treatment for chronic phase $\mathrm{CML}^{13}$ and for gastrointestinal stromal tumor, and it improved average survival from 18 
de Souza et al.

to 57 months. ${ }^{14}$ However, the enormous impact of this and other breakthroughs resulted in a new set of concerns: at prices exceeding $\$ 4500$ per monthalthough justified by high clinical value and costs of innovation-imatinib is a high-value drug that may not be accessible to all patients. ${ }^{15}$ The goal of V-BID in oncology would be to reduce financial barriers to selected services (e.g., evidence-based screening programs) and specific indications (e.g., imatinib in CML), and possibly to extend the concept to the venue of care (e.g., the CalPERS colonoscopy policy), to ultimately increase access and adherence to high-value cancer services.

\section{Evidence-Based Oncology Care: Defining Value Through Evidence}

Oncology interventions differ in the benefits they provide, and the benefit of the same service may have markedly variable clinical effects depending on the population to which it is provided. For example, considering different doses and schedules of administration, bevacizumab is the same price per milligram for patients with metastatic colorectal cancer $(5 \mathrm{mg} / \mathrm{kg}$ every 2 weeks), in whom it provides a median overall survival benefit of 4.7 months ${ }^{16}$ (hazard ratio [HR] for death, 0.66 ; 95\% CI, 0.54-0.81; $P<.001$ ), as it is for those with advanced non-small cell lung cancer (NSCLC; $15 \mathrm{mg} / \mathrm{kg}$ every 3 weeks), in whom it provides a median overall survival benefit of 2 months ${ }^{17}$ (HR, 0.79; 95\% CI, 0.67-0.92; P = .003). It further has the same price per milligram when prescribed for metastatic breast cancer ( $10 \mathrm{mg} / \mathrm{kg}$ every 2 weeks), for which it initially provided a debatable median progression-free survival benefit of 5.5 months ${ }^{18,19}$ (HR for progression, 0.48; 95\% CI, 0.39-0.61; $P<.0001)$ that has not been confirmed by other clinical trials ${ }^{20,21}$ and has no further evidence of improvement in overall survival or quality of life nor FDA approval. This "one size fits all” approach to drug pricing and patient cost-sharing is incongruent in oncology, where a specific intervention has different benefits based on the cancer it is given for or even based on the clinical stage of the disease.

Oncology interventions also differ in their level of supporting evidence. Evidence supporting the duration of adjuvant endocrine therapy with aromatase inhibitors (AIs) for women with hormone receptorpositive breast cancer is a prime example. Although some data support AI use for 5 years based on randomized clinical trials, evidence is still unclear beyond this duration, and 2 ongoing studies are assessing outcomes for longer periods. ${ }^{22,23}$ Accordingly, ASCO recommends AI therapy not to extend beyond 5 years in either the primary or extended adjuvant setting, outside of clinical trials. ${ }^{24}$ Similarly, the NCCN Clinical Practice Guidelines in Oncology (NCCN Guidelines) for Breast Cancer note a category 1 recommendation (high-level of evidence; uniform NCCN consensus) for AI use for 5 years and a category 2B (lower-level of evidence and nonuniform NCCN consensus) for longer periods. ${ }^{25}$

Furthermore, establishing which therapies have high value for oncology patients-ideally based on comparative effectiveness studies-and incorporating this level of evidence into a reimbursement method is a key required step in promoting evidence-based oncology care. Following the earlier examples, one would imagine lower copayments for bevacizumab in metastatic colorectal cancer than in metastatic NSCLC (in which the benefit is quantitatively lower) and metastatic breast cancer (in which the potential benefit is qualitatively different, measured in progression-free survival as opposed to overall survival, and it is not FDA approved). Similarly, copayments for AIs would be lower for 5 years for women with hormone receptor-positive breast cancer and higher after this outside a clinical trial, because evidence is still unclear for longer treatment durations. In other words, V-BID promotes use of interventions with a higher level of evidence and those that provide better outcomes, while discouraging use of off-evidence interventions or those that provide marginal benefit. In addition, through increasing adherence to evidence, guidelines, and high-value interventions, V-BID could potentially curb costs, as decreased use of offevidence therapies and nondrug health care services (e.g., emergency care costs secondary to nonadherence) may offset the additional costs associated with greater use of high-value interventions. ${ }^{26}$

\section{V-BID and Innovation: Promoting Personalized Medicine and Comparative Effectiveness Research}

Trastuzumab is an FDA-approved drug for women with known Her2/neu-positive advanced and earlystage breast cancer. In the United States, up to $20 \%$ 
of patients receiving trastuzumab were not tested for and had no documentation of a positive Her $2 /$ neu test result. ${ }^{27}$ Therefore, these patients were potentially receiving the drug without supporting evidence of value.

Another instance in which patients most likely to benefit from therapy were selected using biomarker analysis, which improved the value of the drug, is gefitinib in NSCLC. An initial clinical trial showed promising results in some patients, ${ }^{28}$ and gefitinib received marketing approval via the accelerated approval pathway. However, subsequent randomized trials failed to confirm its clinical benefit, and marketing approval (for new patients) was withdrawn. Recently, advances in pharmacogenomics have led to the recognition that activating epidermal growth factor receptor (EGFR) mutations identify a new disease subset and individuals who are likely to benefit from treatment with gefitinib. ${ }^{29,30}$ These results prompted the European regulatory authorities to license gefitinib for use in patients with advanced NSCLC who have an activating EGFR mutation. VBID, by varying reimbursements based on available evidence and different values, would promote use of trastuzumab in patients with Her2/neu-positive metastatic breast cancer and gefitinib in patients with NSCLC who have an activating EGFR mutation (through lower copayments) compared with use in patients who are either not tested or who would not benefit from the drug.

In addition, explicitly tying clinician reimbursement/benefit design for an intervention to its survival benefit and evidence adds incentive for conducting comparative effectiveness studies. For example, studies to determine which patient subgroups are most likely to benefit from an intervention or delivery method (e.g., lower doses of the drug, different schedules) would be encouraged early in drug development to increase the value of the intervention.

\section{Next Steps for V-BID in Oncology}

Although V-BID is still novel in oncology, several barriers to implementation should be addressed by the involved stakeholders and policymakers. The high out-of-pocket price for some interventions may inhibit their use by lower-income patients, creating an equity issue. V-BID can potentially reduce the welldescribed socioeconomic disparities in health care delivery, because the effect of patient cost-sharing is inversely related to patient income. Conversely, only allowing interventions to be fully reimbursed if their cost did not exceed a high threshold may result in decreased access to interventions for which there is only marginal or no benefit.

Another potential gap involves indications that fall into the so-called gray zone of evidence-based medicine, within which high-level evidence is difficult to reach even for treatments that are probably effective. Rare and pediatric malignancies, for which large phase III clinical trials are not feasible, fit into this category.

Furthermore, in the United States, discussions among stakeholders and studies to define different patients' preferences and willingness to pay would be needed. In this regard, a recent small pilot survey of cancer survivors undergoing surveillance after curative therapy for localized cancer at a tertiary cancer center suggested that patients may be less willing to pay high copayments for treatments with modest benefits. $^{31}$

When put into context with other novel initiatives that aim to increase quality while decreasing costs in oncology, V-BID addresses different problems in the oncology reimbursement system. For example, the bundling or episode-of-care payment method, piloted by UnitedHealthcare, creates a payment that is based on actual costs plus a case management fee. ${ }^{32}$ This system reduces the conflict of interest that may occur when the provider prescribing the treatment is also the one obtaining financial benefits from the prescription of the treatment, but still does not address the issue of individually high-cost, low-value interventions that may be covered within the bundle. It also rewards and shifts the gains of potential savings to providers, not involving patients in the discussion. On the other hand, the accountable care organization (ACO) concept outlined in the PPACA has the potential to improve coordination of care and also reward providers for decreasing costs while improving quality. ${ }^{33}$ However, as stated by ASCO's provisional opinion, few of the quality measures in the $\mathrm{ACO}$ concept address the nuances of cancer care. ${ }^{34}$ Furthermore, V-BID would complement and add to those novel strategies through directly rewarding patients and shifting interventions to higher quality (and possibly lower cost) care, while maintaining patient and provider autonomy. 
de Souza et al.

\section{Conclusions}

In summary, V-BID is an approach to better align patients' out-of-pocket contributions with the value of an oncology service. Beyond strict cost containment, V-BID in oncology would consist of flexible rules that aim to selectively encourage and reward use of high-value interventions. It would ensure that patients have access to interventions with a high level of evidence or high effectiveness (e.g., screening services and imatinib for CML), while further promoting evidence-based medicine and innovation in oncology. The goal of this clinically nuanced approach is to mitigate underuse of high-value medical services caused by the "one size fits all" cost-sharing model currently used in oncology care in the United States. Through reducing barriers to high-value oncology services across the continuum of cancer care (through lower costs to patients) and discouraging low-value services (through higher costs to patients), these plans can achieve improved health outcomes at any level of health care expenditure.

\section{References}

1. Weaver KE, Rowland JH, Bellizzi KM, Aziz NM. Forgoing medical care because of cost: assessing disparities in healthcare access among cancer survivors living in the United States. Cancer 2010;116:3493-3504.

2. Bernard DS, Farr SL, Fang Z. National estimates of out-of-pocket health care expenditure burdens among nonelderly adults with cancer: 2001 to 2008. J Clin Oncol 2011;29:2821-2826.

3. Mahoney JJ. Reducing patient drug acquisition costs can lower diabetes health claims. Am J Manag Care 2005;11(5 Suppl):S170 S176.

4. Bunting BA, Cranor CW. The Asheville Project: long-term clinical, humanistic, and economic outcomes of a communitybased medication therapy management program for asthma. J Am Pharm Assoc 2006;46:133-147.

5. Agency for Healthcare Research and Quality: Glossary of terms. Available at: http://www.effectivehealthcare.ahrq.gov/index. $\mathrm{cfm} /$ glossary-of-terms/?pageaction=showterm\& amp;termid $=95$. Accessed October 1, 2011.

6. Willey VJ, Pollack MF, Lawless GD. Oncology health care and patient out-of-pocket cost trends in a commercially insured population [abstract]. J Clin Oncol 2008;26(Suppl 15):Abstract 6503.

7. America's Health Insurance Plans (AHIP) Center for Policy and Research. 2011 HSA Census. Available at: http://www. ahipresearch.org/pdfs/HSA2011.pdf. Accessed July 15, 2011.

8. Wharam JF, Graves AJ, Landon BE, et al. Two-year trends in colorectal cancer screening after switch to a high-deductible health plan. Med Care 2011;49:865-871.

9. Jemal A, Bray F, Center MM, et al. Global cancer statistics. CA Cancer J Clin 2011;61:69-90.
10. Holden DJ, Harris R, Porterfield DS, et al. Enhancing the use and quality of colorectal cancer screening. Agency for Healthcare Research and Quality, Rockville, MD. Available at: www.ahrq.gov/ downloads/pub/evidence/pdf/crcuse/crcuse.pdf. Acessed October 2, 2011.

11. Blue Cross Blue Shield of California. Request for information regarding value-based insurance design in connection with preventive care benefits. Available at: http://www.dol.gov/ebsa/pdf/ preventivecare-0037.pdf. Accessed October 1, 2011.

12. Schwartzberg LS, Streeter SB, Husain N, Johnsrud M. Abandoning oral oncolytic prescriptions at the pharmacy: patient and health plan factors influencing adherence [abstract]. J Clin Oncol 2011;29(Suppl 15):Abstract 6036.

13. O'Brien SG, Guilhot F, Larson RA, et al. Imatinib compared with interferon and low-dose cytarabine for newly diagnosed chronicphase chronic myeloid leukemia. N Engl J Med 2003;348:9941004.

14. Blanke CD, Demetri GD, von Mehren M, et al. Long-term results from a randomized phase II trial of standard- versus higher-dose imatinib mesylate for patients with unresectable or metastatic gastrointestinal stromal tumors expressing KIT. J Clin Oncol 2008;26:620-625.

15. Kelley RK, Venook AP. Nonadherence to imatinib during an economic downturn. N Engl J Med 2010;363:596-598.

16. Hurwitz H, Fehrenbacher L, Novotny W, et al. Bevacizumab plus irinotecan, fluorouracil, and leucovorin for metastatic colorectal cancer. N Engl J Med 2004;350:2335-2342.

17. Sandler A, Gray R, Perry MC, et al. Paclitaxel-carboplatin alone or with bevacizumab for non-small-cell lung cancer. N Engl J Med 2006;355:2542-2550.

18. Miller K, Wang M, Gralow J, et al. Paclitaxel plus bevacizumab versus paclitaxel alone for metastatic breast cancer. N Engl J Med 2007;357:2666-2676.

19. Gray R, Bhattacharya $S$, Bowden $C$, et al. Independent review of E2100: a phase III trial of bevacizumab plus paclitaxel versus paclitaxel in women with metastatic breast cancer. J Clin Oncol 2009;27:4966-4972.

20. Miles DW, Chan A, Dirix LY, et al. Phase III study of bevacizumab plus docetaxel compared with placebo plus docetaxel for the first-line treatment of human epidermal growth factor receptor 2-negative metastatic breast cancer. J Clin Oncol 2010;28:32393247.

21. Robert NJ, Dieras V, Glaspy J, et al. RIBBON-1: randomized, double-blind, placebo-controlled, phase III trial of chemotherapy with or without bevacizumab for first-line treatment of human epidermal growth factor receptor 2-negative, locally recurrent or metastatic breast cancer. J Clin Oncol 2011;29:1252-1260.

22. US National Institutes of Health. Letrozole in treating women with primary breast cancer who have received 5 years of aromatase inhibitor therapy. Available at: http://clinicaltrials.gov/ct2/show/ NCT00754845. Accessed October 15, 2011.

23. US National Institutes of Health. Letrozole in treating women who have received hormone therapy for hormone receptorpositive breast cancer. Available at: http://clinicaltrials.gov/show/ NCT00382070. Accessed October 15, 2011.

24. Burstein HJ, Prestrud AA, Seidenfeld J, et al. American Society of Clinical Oncology Clinical Practice Guideline: update on adjuvant endocrine therapy for women with hormone receptor-positive breast cancer. J Clin Oncol 2010;28:3784-3796.

25. Carlson RW, Allred DC, Anderson BO, et al. Invasive breast cancer. J Natl Compr Cancer Netw 2011;9:136-222. 
26. Chernew ME, Juster IA, Shah M, et al. Evidence that value-based insurance can be effective. Health Aff (Millwood) 2010;29:530536.

27. Phillips KA, Marshall DA, Haas JS, et al. Clinical practice patterns and cost effectiveness of human epidermal growth receptor 2 testing strategies in breast cancer patients. Cancer 2009;115:5166-5174.

28. Kris MG, Natale RB, Herbst RS, et al. Efficacy of gefitinib, an inhibitor of the epidermal growth factor receptor tyrosine kinase, in symptomatic patients with non-small cell lung cancer: a randomized trial. JAMA 2003;290:2149-2158.

29. Lynch TJ, Bell DW, Sordella R, et al. Activating mutations in the epidermal growth factor receptor underlying responsiveness of nonsmall-cell lung cancer to gefitinib. N Engl J Med 2004;350:21292139.

30. Mok TS, Wu YL, Thongprasert S, et al. Gefitinib or carboplatinpaclitaxel in pulmonary adenocarcinoma. $N$ Engl J Med 2009;361:947-957.
31. Wong YN, Hamilton $\mathrm{O}$, Egleston $\mathrm{B}$, et al. Understanding how outof-pocket expenses, treatment value, and patient characteristics influence treatment choices. Oncologist 2010;15:566-576.

32. Newcomer LN. Trying something new: episode payments for cancer therapy. J Oncol Pract 2011;7(Suppl 3):S60-61.

33. Centers for Medicare \& Medicaid Services. Medicare Program; Medicare Shared Savings Program: Accountable Care Organizations. 76 Federal Register 19528 (April 7, 2011). Available at: http://edocket.access.gpo.gov/2011/pdf/2011-7880. pdf. Accessed September 7, 2011.

34. American Society of Clinical Oncology. ASCO calls for attention to cancer patients in new delivery models. Available at: http:// www.asco.org/ASCOv2/Department\%20Content/Cancer\%20 Policy\%20and\%20Clinical\%20Affairs/Downloads/ASCO\%20 ACO\%20Comment\%20Letter\%2006-2011.pdf. Accessed October 7, 2011. 